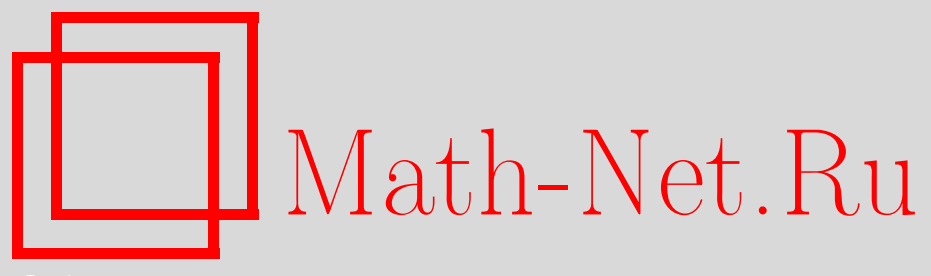

И. В. Артамкин, Ортогональная двойственность торических многообразий Фано с регулярной инволюцией, УМН, 2006, том 61, выпуск 3, 165-166

DOI: https://doi.org/10.4213/rm1698

Использование Общероссийского математического портала Math-Net.Ru подразумевает, что вы прочитали и согласны с пользовательским соглашением http://www . mathnet.ru/rus/agreement

Параметры загрузки:

IP : 34.239 .49 .27

26 апреля 2023 г., $17: 48: 12$

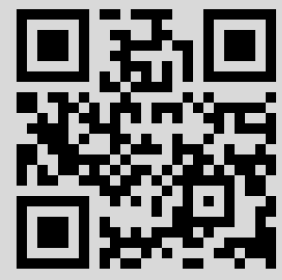




\section{Ортогональная двойственность торических многообразий Фано с регулярной инволюцией}

\section{И. В. Артамкин}

Согласно [1], торические горенштейновы многообразия Фано соответствуют рефлексивным многогранникам. Наличие регулярной инволюции означает, что соответствующий многогранник является центрально-симметричным. Для таких многогранников нам потребуется следующее усиление понятия рефлексивности. Как показано в [1], внутри рефлексивного многогранника (и двойственного к нему) не содержится целых точек, кроме 0 . Рассмотрим решетку $L \cong \mathbb{Z}^{k}$, линейное пространство $W=L \otimes \mathbb{R}$ и центрально-симметричный ограниченный многогранник $\Delta \subset W$. Обозначим через $\pm \xi_{1}, \pm \xi_{2}, \ldots, \pm \xi_{n}$ ненулевые целые точки двойственного многогранника. Пусть $m_{1}, \ldots, m_{n}$ - положительные целые числа. Тогда многогранник $\Delta$ задается (возможно, избыточной) системой неравенств $\left|\xi_{i}(x)\right| \leqslant 1$. Пусть $m_{1}, \ldots, m_{n}$ - положительные целые числа. Тогда набор $\left(\Delta, m_{1}, \ldots, m_{n}\right)$ будем называть взвешенным рефлексивным многогранником.

Отметим, что любой рефлексивный многогранник можно рассматривать как взвешенный, полагая все веса равными единице. В типичном случае, когда все целые точки двойственного многогранника являются его вершинами, уравнения $\xi_{i}(x)= \pm 1$ являются в точности уравнениями граней $\Delta$ максимальной размерности, а взвешенному многограннику тогда соответствует просто обильный дивизор на многообразии Фано, инвариантный относительно инволюции.

Пусть $\left(\Delta, m_{1}, \ldots, m_{n}\right)$ - взвешенный рефлексивный многогранник, положим $N=$ $\sum_{i=1}^{n} m_{i}$ и рассмотрим отображение граней

$$
\Phi=(\underbrace{\xi_{1}, \ldots, \xi_{1}}_{m_{1}}, \underbrace{\xi_{2}, \ldots, \xi_{2}}_{m_{2}}, \ldots, \underbrace{\xi_{n}, \ldots, \xi_{n}}_{m_{n}}): W \rightarrow \mathbb{R}^{N} .
$$

Тогда многогранник $\Phi(\Delta)$ получается пересечением образа $\Phi(W)$ со стандартным кубом $[-1 ; 1]^{N} \subset \mathbb{R}^{N}$. На $\mathbb{R}^{N}$ имеется стандартное скалярное произведение $\langle\cdot, \cdot\rangle ;$ при этом если $c$ - некоторая целая (т.е. принадлежащая решетке $L$ ) точка на границе многогранника $\Delta$, то $|\Phi(c)|^{2}=\sum m_{i}$, где сумма берется по всем граням, содержащим $c$. В частности, если все $m_{i}=1$, то $|\Phi(c)|^{2}$ равно просто числу граней, содержащих $c$.

Рассмотрим ортогональное дополнение $\Phi(W)^{\perp}$ (относительно спаривания $\langle\cdot, \cdot\rangle$ в $\mathbb{R}^{N}$ ) и многогранник $\Delta^{\perp}=[-1 ; 1]^{N} \cap \Phi(W)^{\perp} \subset \mathbb{R}^{N}$. Тогда $\mathbb{Z}^{N} \cap \Phi(W)^{\perp}$ является полномерной решеткой в $\Phi(W)^{\perp}$.

ГипотезА. Решетка $\mathbb{Z}^{N} \cap \Phi(W)^{\perp}$ порождена векторами $\mathbb{Z}^{N} \cap \Delta^{\perp}$, причем каждая грань стандартного куба $[-1 ; 1]^{N}$ пересекает многогранник $\Delta^{\perp}$.

Другими словами, гипотеза утверждает, что $\Delta^{\perp}$ также является взвешенным рефлексивным многогранником, вложенным в $\mathbb{R}^{N}$ при помощи отображения граней, причем соответствующие веса $m_{i}^{\perp}$ граней равны числу граней стандартного куба $[-1 ; 1]^{N}$, пересекающих данную грань $\Delta^{\perp}$.

Соответствующий взвешенный рефлексивный многогранник $\left(\Delta^{\perp}, m_{1}^{\perp}, \ldots, m_{n^{\perp}}^{\perp}\right)$ будем называть ортогонально-двойственным к $\left(\Delta, m_{1}, \ldots, m_{n}\right)$.

Обширный класс примеров ортогонально-двойственных пар взвешенных рефлексивных многогранников получается из теории графов. Для графа $Г$ с множеством вершин $V(|V|=M)$ и множеством ребер $E(|E|=N)$ рассмотрим пространства $\mathbb{R}^{N}=\left\{\sum_{e \in E} \alpha_{e} \cdot e, \alpha_{e} \in \mathbb{R}\right\}$ и $\mathbb{R}^{M}=\left\{\sum_{v \in V} \beta_{v} \cdot v, \beta_{v} \in \mathbb{R}\right\}$, зафиксируем произвольным образом ориентацию на ребрах графа $\Gamma$ и определим граничный и кограничный гомоморфизмы $\partial: \mathbb{R}^{N} \rightarrow \mathbb{R}^{M}$ и $\delta: \mathbb{R}^{M} \rightarrow \mathbb{R}^{N}$, заданные на образующих обычными формулами: $\delta($ вершины $v)=\sum[$ ребер, исходящих из вершины $v]$ и 
$\partial($ ориентированного ребра $e)=[$ конец ребра $e]-[$ начало ребра $e] . \mathrm{B} \mathbb{R}^{N}$ имеется стандартное скалярное произведение $\langle\cdot, \cdot\rangle$, относительно которого $\partial$ и $\delta$ сопряжены, поэтому подпространство ииклов $W=\operatorname{Ker} \partial$ и подпространство разрезов $W^{\perp}=\operatorname{Im} \delta$ представляют собой ортогональное разложение $\mathbb{R}^{N}$. Цикл (разрез) $\sum_{e \in E} \alpha_{e} \cdot e$ называется простым, если все $\alpha_{e}=0, \pm 1$. Многогранник простых ииклов $\Delta(\Gamma)$ - это выпуклая оболочка простых циклов в $W$, многогранник простых разрезов $\Delta^{\perp}(\Gamma)-$ это выпуклая оболочка простых разрезов в $W^{\perp}$.

Теорема 1. 1) Многогранник простых ииклов (разрезов) 2-связного графа Г является рефлексивным многогранником, причем каждому ребру графа соответствует пара граней максимальной размерности, вершинами которой являются простые цикль (разрезы), содержащие данное ребро. При этом все ненулевые целье точки двойственного многогранника являются его вершинами.

2) Многогранники простых ииклов и простых разрезов 2-связного графа Г, в которых каждая грань взята с кратностью, равной числу ребер, которым она соответствует, являются ортогонально-двойственными взвешенными сильно рефлексивными многогранниками.

3) Для 3-связного графа Г веса всех граней многогранников простых ииклов и разрезов равны 1.

Доказательство по существу сводится к рассуждениям, использованным при доказательстве предложения 2.4 в [2]. Там же торическое многообразие Фано, соответствующее многограннику простых циклов графа $\Gamma$, проинтерпретировано как каноническая компактификация обощенного якобиана приводимой кривой с простейшими особенностями, двойственный граф которой совпадает с Г, а все неприводимые компоненты рациональны. Тем самым вопрос о том, определяется ли граф своим многогранником простых циклов, является дискретным аналогом теоремы Торелли.

ТЕОрема 2. 3-связный граф однозначно определяется своим многогранником простых ииклов.

Для доказательства теоремы указывается явная конструкция востановления графа Г по его многограннику простых циклов $\Delta(\Gamma)$. Согласно теореме 1 , восстанавливается многогранник простых разрезов $\Delta^{\perp}(\Gamma)$, среди вершин которого имеются разрезы вида $\delta(v)$, соответствующие всем вершинам $v$ графа Г. Остается заметить, что среди всех вершин многогранника простых разрезов $\Delta^{\perp}(\Gamma)$ вершины вида $\delta(v)$ характеризуются тем, что многогранник, полученный сечением $\Delta^{\perp}(\Gamma)$ гиперплоскостью, ортогональной направлению на данную вершину, является рефлексивным многогранником, не представляющимся в виде прямого произведения меньших таких многогранников.

В заключение приведем пример рефлексивного многогранника, не получающегося ни из какого графа. Это кубооктаэдр - четырнадцатигранник, двойственный к ромбододекаэдру. Его удобно представлять как выпуклую оболочку точек $\left(x_{1}, x_{2}, x_{3}\right) \in \mathbb{R}^{3}$ таких, что $x_{1}+x_{2}+x_{3}$ четно (т.е. принадлежит решетке $D_{3}$ ) и все $x_{i}$ равны $0, \pm 1$. Он обладает (при единичных весах всех граней) четырехмерным ортогонально-двойственным четырнадцатигранником, имеющим 14 вершин с квадратом 4 и 8 вершин с квадратом 7. (На его ребрах имеется еще 12 целых точек с квадратом 3.)

\section{Список литературы}

[1] V. Batyrev, J. Algebraic Geom., 3:3 (1994), 493-535. [2] И. В. Артамкин, Алгебраическая геометрия. Методы, связи и приложения, Труды МИАН, 246, 2004, 10-19.

И. В. Артамкин (I. V. Artamkin)

Математический институт им. В. А. Стеклова РАН

E-mail: artamkin@mail.ru
Представлено С. К. Ландо Принято редколлегией 02.02 .2006 